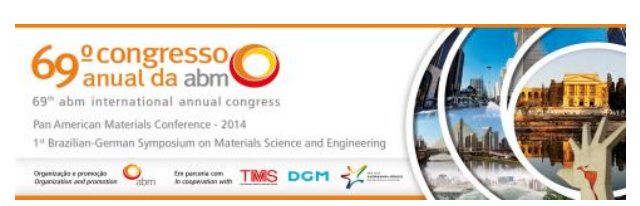

Tema: Iniciação Científico-tecnológica

\title{
ESTIMATIVA DO MÓDULO DE TENACIDADE ATRAVÉS DE INTEGRAÇÃO NUMÉRICA*
}

\author{
Daniel Antonio Kapper Fabricio ${ }^{1}$ \\ Bill Paiva dos Santos ${ }^{2}$ \\ João Vítor Herrmann ${ }^{3}$ \\ Telmo Roberto Strohaecker ${ }^{4}$
}

\begin{abstract}
Resumo
O módulo de tenacidade é um parâmetro que representa a energia absorvida por um material até a ruptura, e é uma variável importante para avaliar se o material sofrerá fratura quando em serviço. A tenacidade é determinada através da área sob a curva tensão-deformação de engenharia, a qual é difícil de ser calculada analiticamente. Assim, o presente trabalho propõe a utilização de métodos numéricos para o cálculo do módulo de tenacidade através de planilhas eletrônicas. Como resultados são apresentados o procedimento desenvolvido e os valores obtidos na estimativa da tenacidade de uma amostra, assim como a comparação do resultado com o valor calculado analiticamente e com o valor calculado através de um método simplificado. Conclui-se que o valor numérico calculado é tão próximo do verdadeiro quanto maior - número de pontos obtidos na curva tensão-deformação de engenharia, viabilizando a utilização desse método na estimativa da tenacidade de uma ampla gama de materiais. Além disso, o método numérico pode ser usado na determinação de outras propriedades calculadas através da área sob curvas.
\end{abstract}

Palavras-chave: Cálculo numérico; Integração numérica; Módulo de tenacidade; Ensaio de tração.

\section{ESTIMATING TENSILE TOUGHNESS THROUGH NUMERICAL INTEGRATION}

\begin{abstract}
The toughness modulus is a parameter that represents the energy absorbed by a material until failure, and is an important variable to assess whether the material will suffer fractures while in operation. The toughness is determined by the area under the engineering stress-strain curve, which is difficult to be calculated analytically. Thus, this paper aims to use numerical methods to estimate the toughness modulus through spreadsheets. As result, there are presented the procedure used and the values obtained in the estimation of the tenacity of a sample, comparing this result with the value calculated by the analytical method and with the value estimated by a simplified method. It is concluded that the numerical value is calculated as close to true as the greatest number of points obtained in the engineering stress-strain curve, enabling the use of this method to estimate the toughness of a wide range of materials. Furthermore, the numerical method can be used to determine other properties calculated by areas under curves.
\end{abstract}

Keywords: Numerical calculus, Numerical integration; Tensile toughness; Tensile test.

Eng. de Produção, Laboratório de Metalurgia Física, UFRGS, Porto Alegre, RS, Brasil. Eng. Metalúrgico, Laboratório de Metalurgia Física, UFRGS, Porto Alegre, RS, Brasil.

Acad. Eng. Mecânica, Laboratório de Metalurgia Física, UFRGS, Porto Alegre, RS, Brasil.

Eng. Metalúrgico, Professor Doutor, PPGE3M, UFRGS, Porto Alegre, RS, Brasil.

\footnotetext{
* Contribuição técnica ao $69^{\circ}$ Congresso Anual da ABM - Internacional e ao 14ํㅡㄹ ENEMET - Encontro Nacional de Estudantes de Engenharia Metalúrgica, de Materiais e de Minas, 21 a 25 de julho de 2014, São Paulo, SP, Brasil.
} 


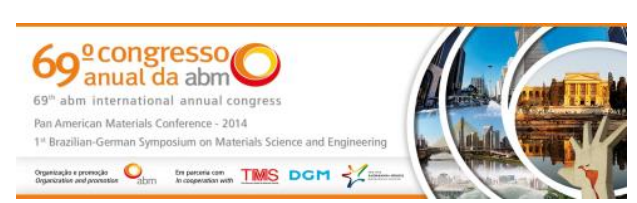

\section{INTRODUÇÃO}

A tenacidade corresponde à capacidade que um material apresenta de absorver energia até a ruptura quando submetido a forças trativas, quantificando a dificuldade ou facilidade de fraturar o material.

Segundo Callister [1], para condições de carregamento dinâmico (a taxas elevadas) e com a presença de concentrador de tensões (entalhe), a tenacidade é calculada através de ensaio de impacto. Para analisar a resistência do material na presença de trincas, calcula-se a tenacidade à fratura. Por fim, na situação estática (baixa taxa de deformação), a tenacidade pode ser apurada a partir dos resultados da área sob a curva tensão-deformação.

A tenacidade para a situação estática pode ser quantificada através do módulo de tenacidade, que consiste na energia absorvida por unidade de volume em um ensaio de tração, podendo ser representada pela área sob a curva tensão-deformação $\left(\sigma \times{ }^{\circ} \varepsilon\right)$ de engenharia [2], definida pela Equação 1. É um parâmetro importante para avaliar se um dado material sofrerá fratura quando em serviço.

$$
U_{T}=\hat{A} r e a_{\sigma \varepsilon}=\int_{\varepsilon_{0}}^{\varepsilon_{f}} \sigma(\varepsilon) d \varepsilon
$$

Note-se que a curva tensão-deformação de engenharia, plotada a partir do ensaio de tração, considera que a área da seção do corpo de prova se mantém constante durante o ensaio, ignorando a redução de área que ocorre durante o ensaio. Em algumas aplicações, pode ser importante considerar a curva tensão-deformação verdadeira, a qual considera a tensão como relação entre a força instantânea e a área instantânea [1].

A ausência de uma expressão analítica que represente a tensão $(\sigma)$ como função da deformação $(\varepsilon)$, dificulta o cálculo da área sob a curva tensão-deformação de engenharia e, consequentemente, a determinação do módulo de tenacidade (UT) [1]. A literatura [2,3] apresenta um método simplificado para a estimativa do módulo de tenacidade. Caso se deseje uma maior precisão no cálculo da tenacidade, podem ser utilizados métodos numéricos para esse cálculo.

Ressalta-se que, nesse trabalho, o cálculo do módulo de tenacidade foi baseado na área sob a curva tensão-deformação de engenharia, e não na curva verdadeira. Não foi possível calcular a tenacidade para a curva verdadeira, pois a estricção invalida as equações para conversão da curva verdadeira em relação à de engenharia. Apesar dessa limitação, os métodos numéricos podem utilizar com sucesso os dados de engenharia nesse tipo de análise, sendo esse o principal objetivo do presente trabalho.

\section{MATERIAIS E MÉTODOS}

Para a execução desse trabalho, foram seguidas as seguintes etapas:

- Execução de um ensaio de tração e plotagem da curva tensão-deformação;

- Cálculo da tenacidade numericamente;

- Cálculo da tenacidade analiticamente;

- Cálculo da tenacidade por um método simplificado.

O ensaio de tração foi executado em uma máquina eletromecânica marca Instron modelo $5585 \mathrm{H}$ em um aço baixa liga. Em seguida, foi plotada a curva $\sigma \times \varepsilon$, a partir da qual foi calculado o módulo de tenacidade utilizando o método numérico da Regra dos Trapézios. A Regra dos Trapézios consiste em se aproximar o valor de

\footnotetext{
* Contribuição técnica ao $69^{\circ}$ Congresso Anual da ABM - Internacional e ao 14ํㅡㄹ ENEMET - Encontro Nacional de Estudantes de Engenharia Metalúrgica, de Materiais e de Minas, 21 a 25 de julho de 2014, São Paulo, SP, Brasil.
} 


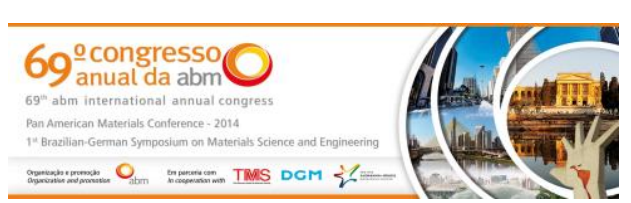

uma função contínua de $f(x)$ no intervalo $\left[x_{0}, x_{1}\right]$ por uma função de primeira ordem. Isto, geometricamente, é equivalente a aproximar uma curva qualquer por uma reta. Desta forma, a área sob a função $f(x)$ é aproximada pela área do trapézio cuja largura é igual a $\left(x_{1}-x_{0}\right)$ e a altura média igual a $\left[f\left(x_{0}\right)+f\left(x_{1}\right)\right] / 2$ (Equação 2).

$$
\text { Área }=\int_{x_{0}}^{x_{1}} f(x) d x \cong\left(x_{1}-x_{0}\right) \frac{\left[f\left(x_{0}\right)+f\left(x_{1}\right)\right]}{2}
$$

Para um conjunto de $n$ pontos do tipo $\left(x_{i}, f\left(x_{i}\right)\right)$, para $a \leq x_{i} \leq b$, uma boa aproximação para a área sob a curva entre a e b é a soma dos n-1 trapézios sob a curva, isto é:

$$
\text { Área }=\int_{a}^{b} f(x) d x \cong \sum_{i=1}^{n-1}\left(x_{i+1}-x_{i}\right) \frac{\left[f\left(x_{i}\right)+f\left(x_{i+1}\right)\right]}{2}
$$

A Figura 1 esquematiza o cálculo da área através desse método, utilizando $n=6$ pontos (5 trapézios). Note-se que, quanto maior o número de pontos $\left(x_{n}, f\left(x_{n}\right)\right)$ utilizados no cálculo, mais a integral numérica se aproximará do valor verdadeiro.

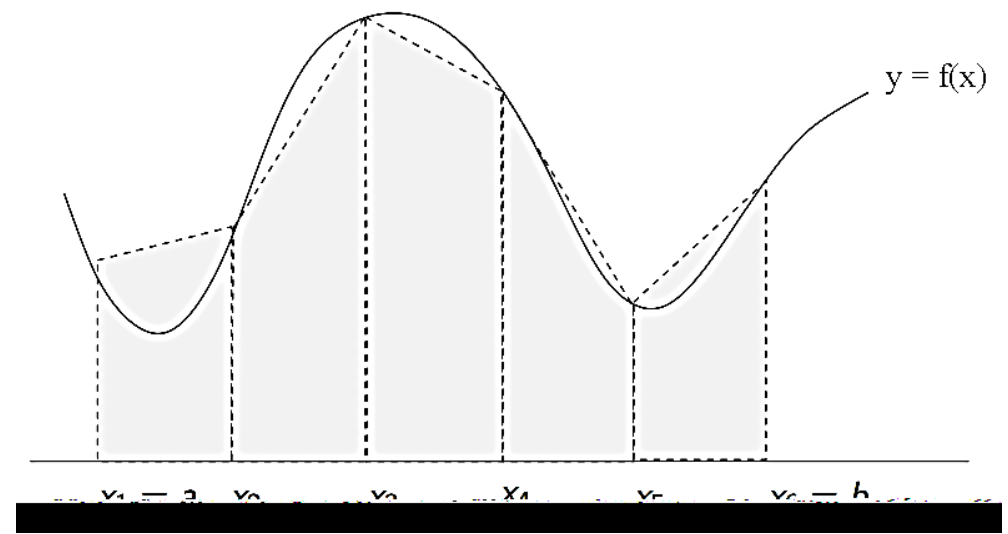

Figura 1. Regra dos trapézios [4].

Após calcular o módulo de tenacidade pela Regra dos Trapézios, essa propriedade foi calculada, para efeitos comparativos, através de análise de regressão da curva tensão-deformação. Para isso, a curva foi dividida em duas partes: na região elástica, foi utilizada regressão linear, e na região plástica, foi utilizada regressão polinomial através de um polinômio de grau 6. A análise da regressão foi realizada através de planilhas eletrônicas. A partir das equações de regressão linear e polinomial, calculou-se a integral de modo analítico, utilizando-se o Cálculo.

Por fim, o mesmo módulo de tenacidade foi estimado através da aproximação descrita por Garcia, Spim e Santos [2] e por Dowling [3], apresentada na Equação 4. Esse método aproxima a área sob a curva tensão-deformação de engenharia a um retângulo, conforme a Figura 2 .

$$
U_{T}=\frac{\sigma_{E}+\sigma_{U}}{2} \varepsilon_{f}
$$

$\mathrm{Na}$ Equação 4, $\sigma_{\mathrm{E}}$ representa a tensão de escoamento, $\sigma \cup \mathrm{o}$ limite de resistência e $\varepsilon f$ a deformação máxima.

Segundo Dowling [3], tal aproximação pode ser realizada quando a curva é relativamente plana além do ponto de escoamento.

\footnotetext{
* Contribuição técnica ao 69ํㅡㄹ Congresso Anual da ABM - Internacional e ao 14 ENEMET - Encontro Nacional de Estudantes de Engenharia Metalúrgica, de Materiais e de Minas, 21 a 25 de julho de 2014, São Paulo, SP, Brasil.
} 

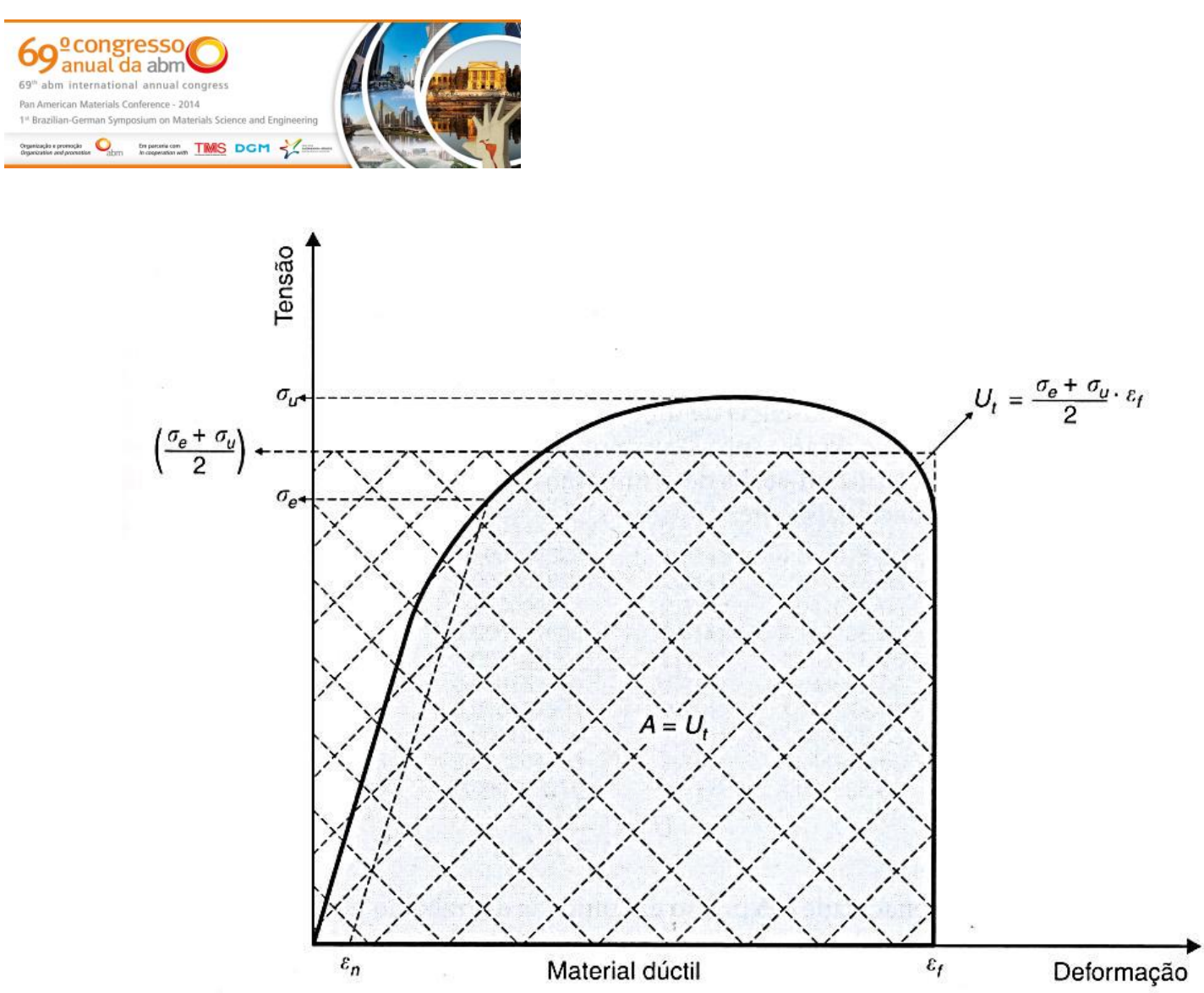

Figura 2. Estimativa do módulo de tenacidade para materiais dúcteis [2].

\section{RESULTADOS E DISCUSSÃO}

\subsection{Ensaio de Tração}

O ensaio de tração foi executado em uma amostra padronizada segundo a norma internacional ASTM E8/E8M [5], utilizando-se uma taxa de deslocamento de 0,45 $\mathrm{mm} / \mathrm{min}$, dentro da faixa de deslocamento sugerida para corpos de prova com comprimento útil (Lo) de $25 \mathrm{~mm}$.

$\mathrm{Na}$ Tabela 1, são apresentadas as propriedades de tração obtidas a partir da curva tensão-deformação de engenharia.

Tabela 1. Resultados obtidos a partir da curva tensão-deformação de engenharia

\begin{tabular}{l|c}
\multicolumn{1}{c|}{ Propriedade } & Valor \\
\hline Deformação Máxima na Fratura $\left(\varepsilon_{\mathrm{f}}\right)$ & $0,16 \mathrm{~mm} / \mathrm{mm}$ \\
\hline Carga de Escoamento $\left(\mathrm{F}_{\mathrm{E}}\right)$ & $19640,77 \mathrm{~N}$ \\
\hline Limite de Escoamento $\left(\sigma_{\mathrm{E}}\right)$ & $980,59 \mathrm{MPa}$ \\
\hline Carga Máxima $\left(\mathrm{F}_{\mathrm{U}}\right)$ & $21661,45 \mathrm{~N}$ \\
\hline Limite de Resistência $\left(\sigma_{\mathrm{U}}\right)$ & $1081,47 \mathrm{MPa}$ \\
\hline Redução de Área $(\mathrm{n})$ & $61,09 \%$ \\
\hline
\end{tabular}

A Figura 3 apresenta a curva tensão-deformação de engenharia obtida no ensaio.

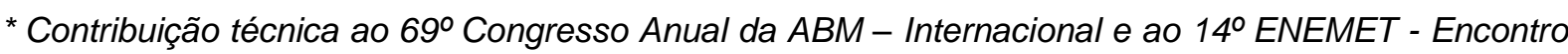
Nacional de Estudantes de Engenharia Metalúrgica, de Materiais e de Minas, 21 a 25 de julho de 2014, São Paulo, SP, Brasil.
} 


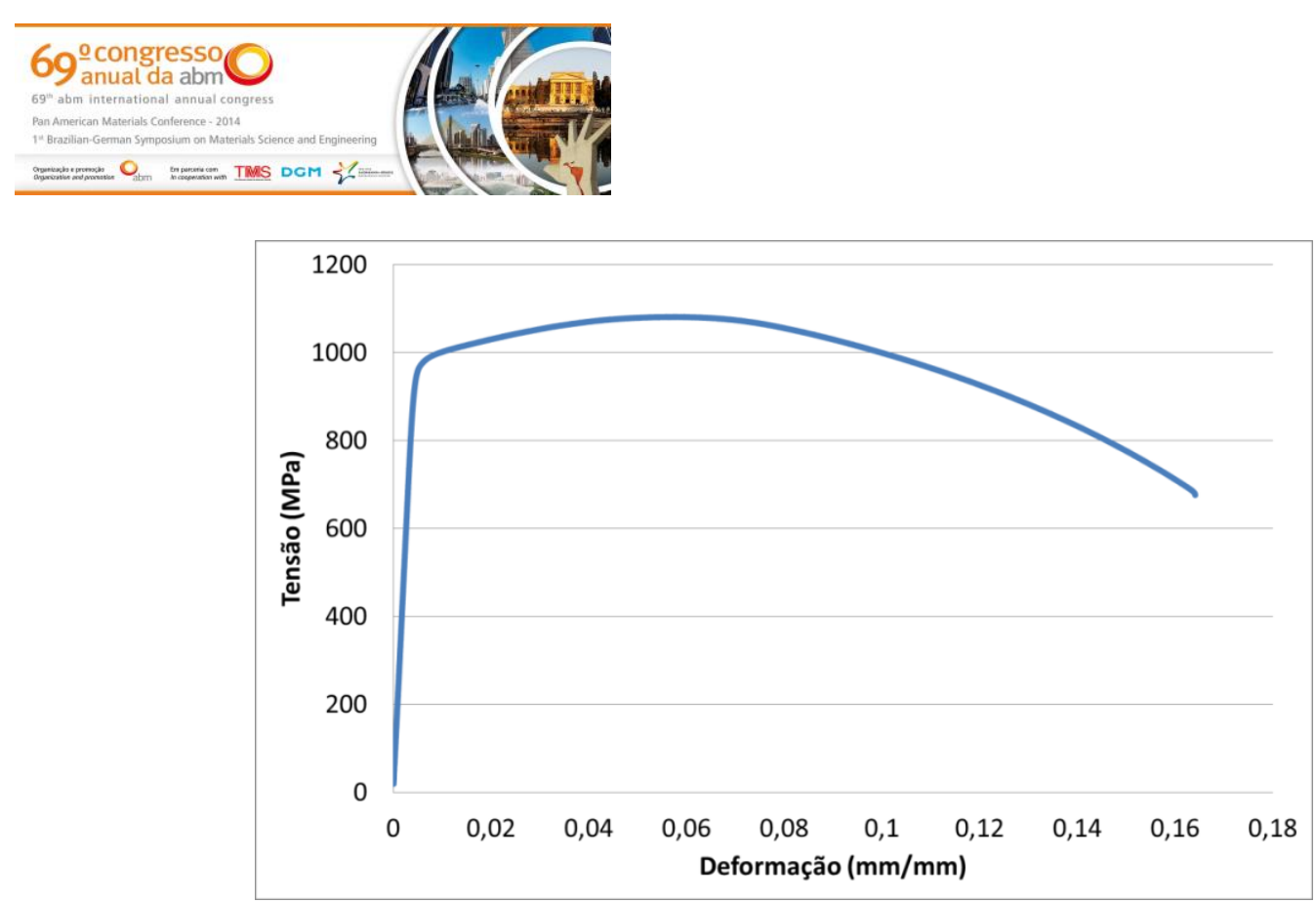

Figura 3. Curva tensão-deformação de engenharia.

\subsection{Cálculo da Tenacidade Numericamente}

A Regra dos Trapézios foi aplicada com os dados obtidos da máquina de ensaio, em planilha eletrônica. Foi gerada a curva tensão-deformação com $n=3116$ pontos. $\mathrm{Ou}$ seja, o número de trapézios usados no cálculo foi de 3115. A Tabela 2 apresenta um trecho da planilha, exemplificando como foi realizado o cálculo do módulo de tenacidade.

Tabela 2. Cálculo da tenacidade numericamente

\begin{tabular}{c|c|c}
\hline $\begin{array}{c}\text { Deformação } \\
(\mathrm{mm} / \mathrm{mm})\end{array}$ & Tensão $(\mathrm{MPa})$ & $\begin{array}{c}\text { Área do n-ésimo } \\
\text { Trapézio }\left(\mathrm{N} . \mathrm{mm} / \mathrm{mm}^{3}\right)\end{array}$ \\
\hline 0,00018338 & 40,82791 & - \\
\hline 0,00018338 & 43,17994 & 0,000000000 \\
\hline 0,00019338 & 45,98937 & 0,000445847 \\
\hline 0,00020338 & 48,43247 & 0,000472109 \\
\hline 0,00022338 & 50,87012 & 0,000993026 \\
\hline 0,00023338 & 53,73606 & 0,000523031 \\
\hline 0,00023338 & 56,7126 & 0,000000000 \\
\hline 0,00024338 & 59,68418 & 0,000581984 \\
\hline 0,00026338 & 62,22595 & 0,001219101 \\
\hline 0,00028338 & 65,13779 & 0,001273637 \\
\hline 0,00029338 & 67,99438 & 0,000665661 \\
\hline 0,00031338 & 71,35196 & 0,001393463 \\
\hline
\end{tabular}

A partir da Tabela 2, o módulo de tenacidade foi calculado através da soma das áreas dos $\mathrm{n}-1$ trapézios. Foi obtido, como resultado, um módulo de tenacidade de 157,82 N.mm $/ \mathrm{mm}^{3}$.

\subsection{Cálculo da Tenacidade Analiticamente}

Para realizar o cálculo do módulo de tenacidade através de integração analítica, foi realizada a regressão da curva tensão-deformação. Como a curva possui uma região elástica e uma região plástica, ela foi dividida em duas partes, para se obter

\footnotetext{
* Contribuição técnica ao $69^{\circ}$ Congresso Anual da ABM - Internacional e ao 14ํㅡㄹ ENEMET - Encontro Nacional de Estudantes de Engenharia Metalúrgica, de Materiais e de Minas, 21 a 25 de julho de 2014, São Paulo, SP, Brasil.
} 


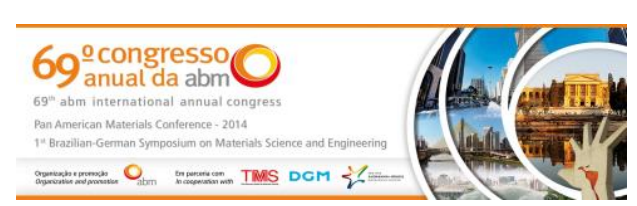

uma melhor estimativa da regressão. Na região elástica, foi realizada regressão linear, e na região plástica, regressão polinomial por um polinômio de grau 6.

A Figura 4 apresenta a curva e a equação de regressão para a região elástica (nesse caso, com $\varepsilon$ variando entre 0,000 e $0,004 \mathrm{~mm} / \mathrm{mm}$ ).

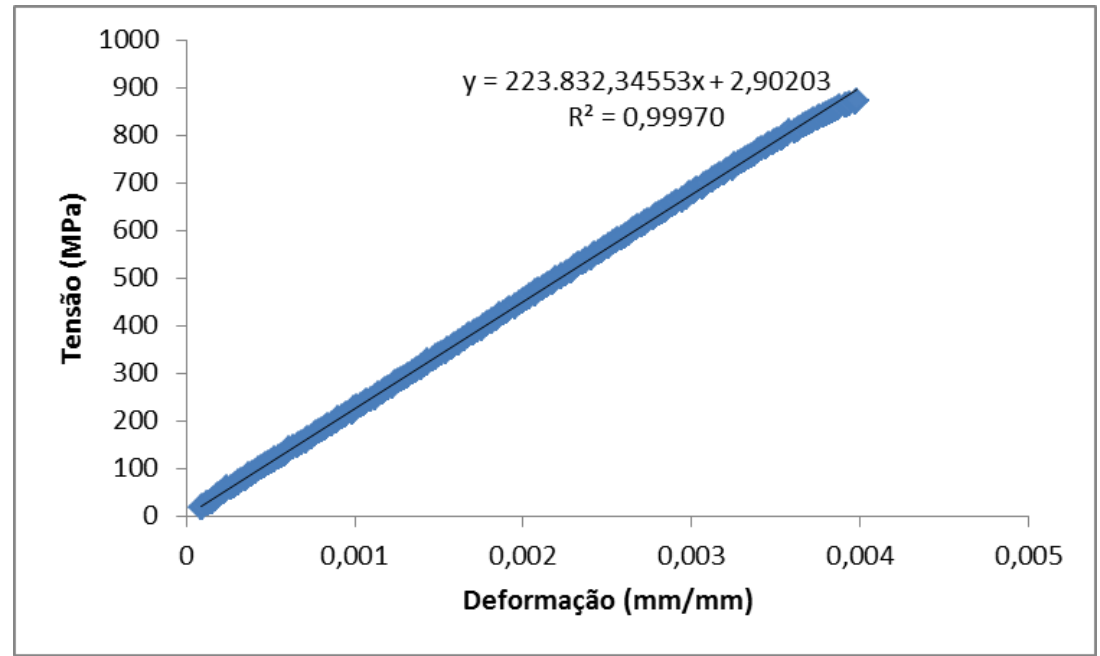

Figura 4. Regressão da região elástica.

A Figura 5 representa a regressão para a região plástica, com $\varepsilon$ variando entre 0,004 $\mathrm{mm} / \mathrm{mm}$ e $0,164 \mathrm{~mm} / \mathrm{mm}$, quando ocorreu a ruptura do corpo de prova.

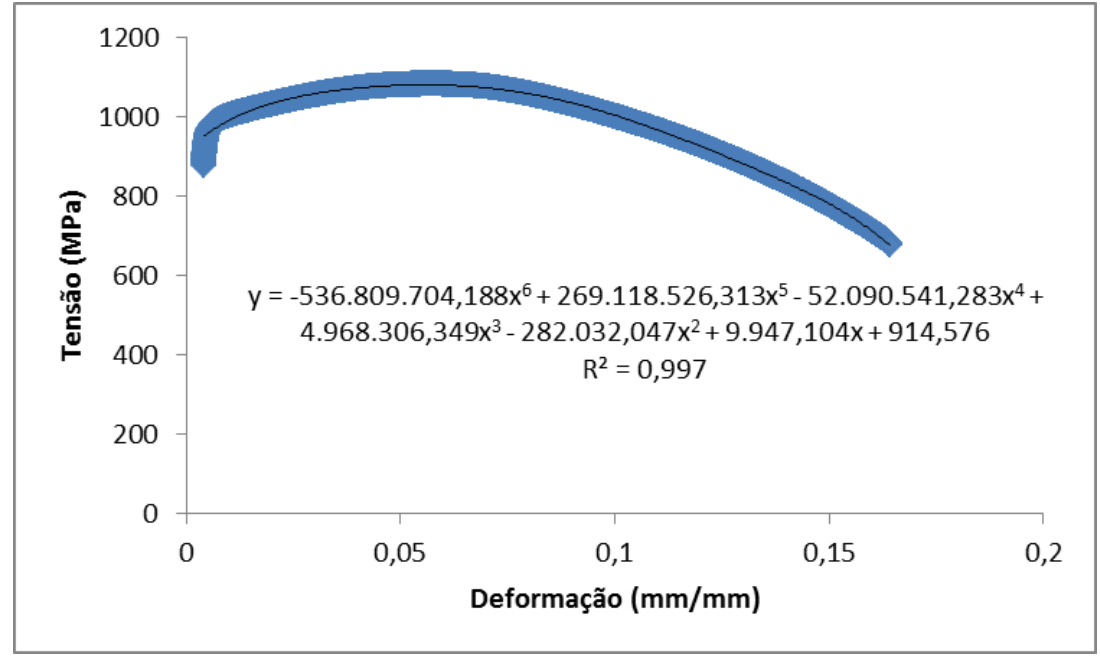

Figura 5. Regressão da região plástica.

O valor de $\varepsilon$ em que o comportamento da curva se altera de elástico para plástico é difícil de ser detectado com precisão. Nesse trabalho, a curva foi analisada ponto a ponto visando estimar o ponto de transição elasto-plástica, sendo encontrado um valor de $\varepsilon$ de aproximadamente $0,004 \mathrm{~mm} / \mathrm{mm}$. Ainda que esse ponto tenha sido localizado por análise gráfica, sem garantir que esse ponto seja preciso, essa escolha mostrou-se suficiente, visto que as curvas de regressão foram plotadas com valores dos coeficientes de determinação $\left(R^{2}\right)$ próximo de 1 . Por definição, isso indica que as regressões calculadas representam uma boa aproximação da curva real.

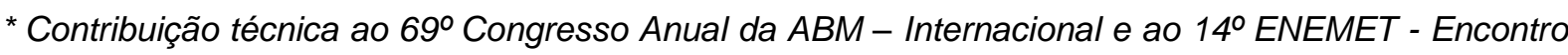
Nacional de Estudantes de Engenharia Metalúrgica, de Materiais e de Minas, 21 a 25 de julho de 2014, São Paulo, SP, Brasil.
} 


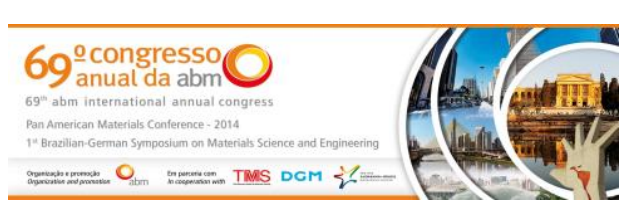

Assim, o módulo de tenacidade UT pode ser aproximado através da soma das áreas sob as duas curvas de regressão, ou seja, a soma das integrais de y ( $\sigma)$ em função de $x(\varepsilon)$ :

$$
\begin{array}{r}
U_{T}=\int_{a}^{b} f(x) d x \cong \int_{0}^{0,004}(223.832,346 \mathrm{x}+2,90203) \mathrm{dx}+\int_{0,004}^{0,164}\left(-536.809 .704,188 x^{6}+\right. \\
269.118 .526,313 x^{5}-52.090 .541,283 x^{4}+4.968 .306,349 x^{3}-282.032,047 x^{2}+ \\
9.947,104 \mathrm{x}+914,576) d x=157,7690 \mathrm{~N} . \mathrm{mm} / \mathrm{mm}^{3}
\end{array}
$$

Pela Equação 5, o valor do módulo de tenacidade obtido pela regressão é de aproximadamente $157,77 \mathrm{~N} \cdot \mathrm{mm} / \mathrm{mm}^{3}$.

\subsection{Cálculo da Tenacidade pelo Método Simplificado}

Aplicando-se a aproximação descrita por Garcia, Spim e Santos [2] e por Dowling [3], (Equação 4), com $\sigma_{E}=980,59 \mathrm{MPa}, \sigma u=1081,47 \mathrm{MPa}$ e $\varepsilon_{f}=0,16 \mathrm{~mm} / \mathrm{mm}$, obteve-se UT $=169,25 \mathrm{~N} \cdot \mathrm{mm} / \mathrm{mm}^{3}$.

\section{DISCUSSÃO}

A Tabela 3 apresenta uma comparação do módulo de tenacidade calculado pelo método numérico (Regra dos Trapézios), em comparação com o método analítico (regressão) e com o método simplificado.

Tabela 3. Comparação dos resultados

\begin{tabular}{c|c|c}
\hline $\begin{array}{c}\text { Método } \\
\text { utilizado }\end{array}$ & $\begin{array}{c}\text { Módulo de tenacidade } \\
\text { calculado }\left(\mathrm{N} . \mathrm{mm} / \mathrm{mm}^{3}\right)\end{array}$ & $\begin{array}{c}\text { Desvio em relaćão ao } \\
\text { método numérico }\end{array}$ \\
\hline Numérico & 157,82 & - \\
\hline Analítico & 157,77 & $-0,03 \%$ \\
\hline Simplificado & 169,26 & $+7,25 \%$ \\
\hline
\end{tabular}

Nota-se que a diferença entre o método numérico e o analítico foi muito baixa. Já a diferença entre o método numérico e o método simplificado é significativamente maior. Isso já era esperado, visto que esse último método é uma aproximação mais grosseira da área sob a curva tensão-deformação.

Como os métodos analítico e numérico resultaram em valores semelhantes, sugerese o uso de algum desses métodos ao invés do método simplificado. Assim, indicase a escolha do método numérico para a determinação do módulo de tenacidade em trabalhos futuros, visto que tal método é mais fácil de aplicar que o analítico, sem a necessidade de resolver a integral e de localizar o ponto de transição elásticaplástica.

No entanto, no caso do método numérico, ressalta-se que a precisão na determinação do módulo de tenacidade é tão boa quanto maior o número de pontos adquiridos no ensaio de tração. Caso o número de pontos seja pequeno, o cálculo será prejudicado.

A Tabela 4 resume as vantagens e limitações de cada método identificadas no decorrer do trabalho.

\footnotetext{
* Contribuição técnica ao $69^{\circ}$ Congresso Anual da ABM - Internacional e ao 14ํㅡㄹ ENEMET - Encontro Nacional de Estudantes de Engenharia Metalúrgica, de Materiais e de Minas, 21 a 25 de julho de 2014, São Paulo, SP, Brasil.
} 


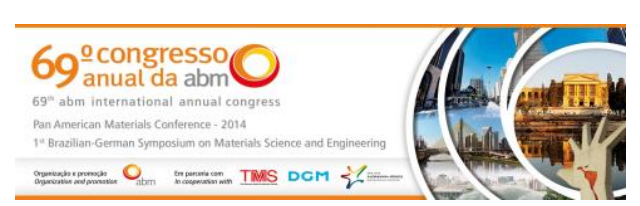

Tabela 4. Comparação entre os métodos

\begin{tabular}{|c|c|c|}
\hline $\begin{array}{l}\text { Método } \\
\text { utilizado }\end{array}$ & Vantagens & Limitações \\
\hline Numérico & 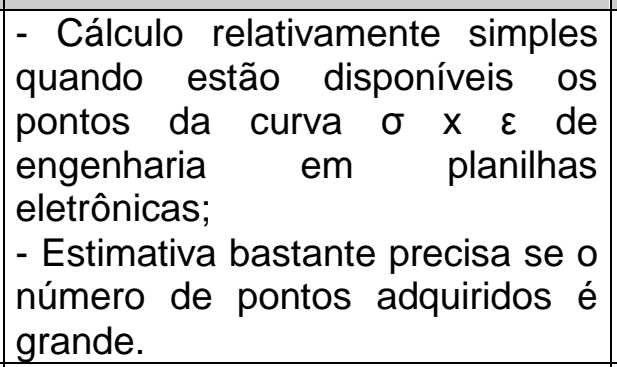 & $\begin{array}{l}\text { - Quanto menor o número de pontos, } \\
\text { menos exato é o cálculo; } \\
\text { - Por se tratar de um método numérico, } \\
\text { tende a ser menos exato que a } \\
\text { integração por Cálculo. }\end{array}$ \\
\hline Analítico & $\begin{array}{l}\text { - Método bastante robusto, pois } \\
\text { segue a definição básica de área } \\
\text { sob a curva a partir do Cálculo; } \\
\text { - Estimativa bastante precisa se o } \\
\text { número de pontos adquiridos é } \\
\text { grande. }\end{array}$ & $\begin{array}{l}\text { - Método mais trabalhoso, visto que há } \\
\text { necessidade de calcular a área via } \\
\text { Cálculo tradicional; } \\
\text { - Dificuldade de determinar o ponto } \\
\text { exato de transição elástica-plástica; } \\
\text { - Caso haja ruído (erros) na aquisição } \\
\text { da curva, o coeficiente de determinação } \\
\text { será menor que 1, ocasionando maior } \\
\text { imprecisão na plotagem da curva de } \\
\text { regressão; }\end{array}$ \\
\hline Simplificado & $\begin{array}{l}\text { - Cálculo bastante simples; } \\
\text { - Dependendo da aplicação, } \\
\text { fornece uma estimativa razoável } \\
\text { de } U_{T} ; \\
\text { - Pode ser utilizado mesmo que o } \\
\text { número de pontos adquiridos seja } \\
\text { pequeno. }\end{array}$ & $\begin{array}{l}\text { - Estimativa bastante grosseira em } \\
\text { comparação com os outros métodos, } \\
\text { principalmente se o comportamento da } \\
\text { curva tensão-deformação de engenharia } \\
\text { não for plano após o escoamento. }\end{array}$ \\
\hline
\end{tabular}

\section{CONCLUSÃo}

No presente trabalho, foi realizado um ensaio de tração com ênfase no cálculo do módulo de tenacidade, utilizando o método numérico da Regra dos Trapézios. Para efeitos comparativos, o módulo de tenacidade foi calculado através da regressão da curva tensão-deformação (seguida da integração analítica), e também utilizando-se a aproximação simplificada dessa propriedade conforme proposto por Garcia, Spim e Santos [2] e por Dowling [3].

Utilizando-se um número relativamente grande de pontos, tanto o método numérico quanto o método analítico apresentaram uma boa estimativa do módulo de tenacidade em comparação com o método simplificado, sendo o método numérico mais fácil de executar a partir dos dados do ensaio de tração.

O método analítico é mais trabalhoso, pois nem sempre é fácil encontrar o ponto de transição entre o regime linear e o regime plástico. Além disso, o uso desse método requer que a área sob as curvas de regressão seja calculada através de integração analítica, o que muitas vezes pode ser difícil.

Assim, em trabalhos futuros, sugere-se o uso do método numérico (Regra dos Trapézios) para o cálculo do módulo de tenacidade, dada a simplicidade de sua aplicação em comparação com o método analítico e a sua maior precisão em comparação com o método simplificado proposto por Garcia, Spim e Santos [2] e por Dowling [3].

\footnotetext{
* Contribuição técnica ao $69^{\circ}$ Congresso Anual da ABM - Internacional e ao 14ํㅡㄹ ENEMET - Encontro Nacional de Estudantes de Engenharia Metalúrgica, de Materiais e de Minas, 21 a 25 de julho de 2014, São Paulo, SP, Brasil.
} 


\section{Agradecimentos}

Os autores agradecem ao CNPq e a Capes pelo incentivo nas atividades de pesquisa e desenvolvimento.

\section{REFERÊNCIAS}

1 Callister WD. Materials science and engineering: an introduction. $7^{\text {a }}$ edição. New York: John Wiley \& Sons; 2007.

2 Garcia A, Spim JA, Santos CA. Ensaios dos Materiais. 2ª edição. Rio de Janeiro: LTC; 2012.

3 Dowling NE. Mechanical Behaior of Materials: Engineering Methos for Deformation, Fracture, and Fatigue. 3ae edição. New Jersey: Pearson Prentice Hall, 2007.

4 Camponogara E. Integração numérica: Introdução, regra dos retângulos e regra do trapézio. Acesso em 06 mar. 2014. Disponível em:

http://www.das.ufsc.br/ camponog/Disciplinas/DAS-5103/Slides/l20-numint-intro.pdf.

5 ASTM E8/EM8. Standard Test Methods for Tension Testing of Metallic Materials. Pennsylvania: ASTM International; 2013.

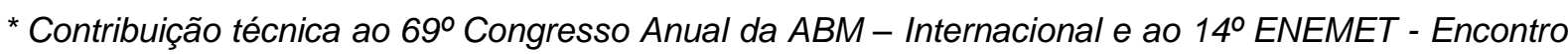
Nacional de Estudantes de Engenharia Metalúrgica, de Materiais e de Minas, 21 a 25 de julho de 2014, São Paulo, SP, Brasil. 